\title{
Prise en charge de l'hémophilie A majeure en pratique odontologique à Dakar : présentation de deux observations
}

\author{
Dia Tine $\mathbf{S}^{\mathbf{1}}$, Barry $\mathrm{BC}^{\mathbf{1}}$, Tamba $\mathbf{B}^{\mathbf{1}}$, Niang $\mathbf{P}^{\mathbf{1}}$, Dia Lissa ${ }^{\mathbf{1}}$, Diop Saliou ${ }^{\mathbf{2}}$, Diallo $\mathrm{B}$ \\ ${ }^{1}$ Département d'Odontologie, Service de Chirurgie buccale, Université Cheikh Anta Diop, \\ Dakar, Sénégal \\ ${ }^{2}$ Centre national de Transfusion sanguine, Dakar, Sénégal \\ diatine@hotmail.com
}

L'hémophilie est caractérisée par un déficit en facteurs de la coagulation : il s'agit du facteur VIII pour l'hémophilie A et du facteur IX pour l'hémophilie B. Seuls les sujets mâles sont atteints car le gène codant pour ces facteurs est situé sur le chromosome X (Bernard 1990). C'est une maladie rare : un nouveau né mâle sur 5000 est atteint. Le diagnostic biologique est posé devant un allongement du TCA associé à un déficit en facteur (Alantar et al. 2005). La prévalence de l'hémophilie $\mathrm{A}$ est de loin supérieure à celle l'hémophilie B au Sénégal avec un taux de $92 \%$ d'hémophiles A pour 8\% d'hémophiles B (Thiam et al. 1997). On distingue trois formes en fonction de l'importance du déficit en facteur. Dans les formes sévères, le taux d'activité du facteur est $<1 \%$, et le diagnostic est en général précoce (Piott 1995, Diallo 2003). L'hémorragie peut revêtir un aspect pathologique dramatique dans les formes sévères qui représentent 40\% des cas au Sénégal (Diallo 2003). Les saignements bucco-dentaires chez l'enfant sont occasionnés le plus souvent par l'éruption des dents ou des traumatismes lors des chutes. Nous rapportons le cas de deux enfants, âgés respectivement de 2 et 3 ans, atteints d'une hémophilie A sévère et ayant présenté des hémorragies importantes de la cavité buccale. La prise en charge thérapeutique a été assurée grâce à la collaboration de différents praticiens hématologistes, pédiatres, anesthésistes, et odontologistes dans un service hospitalier à Dakar. Le protocole d'hémostase adopté est conforme aux critères DCP : adapté au type et à l'intensité du Déficit (D) en facteur de coagulation, au type de Chirurgie $(C)$ et à la compliance du patient (P) (Alantar 2005).

Observation $\mathrm{n}^{\circ} 1$ : D L est un garçon de 2 ans qui est atteint d'une hémophilie A majeure, aggravée par la présence d'anticorps circulants. Il a présenté deux épisodes d'hémorragie en 3 mois. Le premier épisode s'est manifesté par un important saignement en nappe de la gencive lors de l'éruption d'une dent. Le $2^{\mathrm{ème}}$ a été consécutif à une chute à domicile ayant entrainé une plaie punctiforme et pénétrante sur la face ventrale de la pointe de la langue.

Observation $n^{\circ} 2:$ M C est un garçon de 3 ans qui a été victime d'une chute à domicile, ayant entrainé un traumatisme alvéolo-dentaire avec fracture coronaire basse de 51 et 61 , associée à une plaie gingivale très hémorragique.

Dans les 2 cas, le saignement a persisté par intermittence pendant 48 heures malgré la transfusion de facteur VIII et l'utilisation des moyens d'hémostase locale disponibles dans le Service de Pédiatrie. Devant l'impossibilité de pratiquer tout acte sous anesthésie locale chez ces enfants très agités, l'hémostase a été réalisée sous anesthésie générale par coagulation au bistouri électrique, complétée par des sutures et une compression extrinsèque avec des mèches d'oxycellulose ou des compresses imbibées d'antifibrinolitiques (acide tranéxamique). La prescription d'antibiotiques et d'antalgiques a été systématique. L'arrêt total de l'hémorragie a été obtenu en moins de 24 heures.

La prise en charge en chirurgie buccale du saignement chez l'hémophile doit être adaptée à la sévérité de la pathologie et à la gravité du saignement. L'implication de l'odontologiste dans l'équipe pluridisciplinaire doit être mieux considérée dans notre pratique à Dakar. 